\title{
EQUIDISTANT SETS IN PLANE TRIODIC CONTINUA
}

\author{
L. D. LOVELAND AND S. M. LOVELAND
}

(Communicated by James E. West)

\begin{abstract}
Let $x$ and $y$ be two points in a metric space $(X, \rho)$. The equidistant set or midset $M(x, y)$ of $x$ and $y$ is the set $\{p \in X \mid \rho(x, p)=\rho(y, p)\}$. If the midset of each pair of points of $X$ consists of a finite number of points then the metric space $X$ is said to have the finite midset property, and if the midsets of pairs of points in $X$ are pairwise homeomorphic then $X$ is said to have uniform midsets. Generalizing earlier results, the main theorem states that no continuum in the Euclidean plane can have both finite and uniform midsets if it contains a triod. It follows that a plane continuum with finite, uniform midsets must be either an arc or a simple closed curve.
\end{abstract}

\section{INTRODUCTION}

For each positive integer $n$, a metric space $X$ is said to have the $n$-point midset property, abbreviated as the $n$-MP, if for every two points $x$ and $y$ in $X$ the set $M(x, y)$ of all points of $X$ equidistant from $x$ and $y$ consists of $n$ points. Thus a space with finite, uniform midsets has the $n$-MP for some positive integer $n$. A circle or, more generally, any simple closed curve in the Euclidean plane $E^{2}$ that bounds a convex disk has the 2-MP. This 2-MP is more commonly called the double midset property and is denoted by DMP. In [4] it was proved that a continuum in the plane with the DMP must be a simple closed curve, and it has been conjectured [8] that this result is true without restriction to the Euclidean plane $E^{2}$. It was also conjectured in [8] that no continuum has the 3-MP, a property previously called the triple midset property (TMP), and this was also verified for continua in $E^{2}[5,6]$. Theorem 3.1 of this paper states that no continuum in $E^{2}$ that contains a triod can have the $n$-MP for any positive integer $n$. The special cases $n=2$ and $n=3$ of Theorem 3.1 include Theorem 2.1 of [6] and Theorem 2 of [4], respectively. In another paper [7], we show that no arc or simple closed curve in $E^{2}$ can have the $n$-MP for $n>2$.

No examples have been found of continua with the $n$-MP, except for the cases where $n \in\{1,2\}$. Theorem 3.2 states that the only plane continua with

Received by the editors September 7, 1990.

1991 Mathematics Subject Classification. Primary 54F20, 54F50; Secondary 51K05, 54F65.

Key words and phrases. Arc, bisector, continuum, equidistant set, midset, simple closed curve, triod, uniform midset, midset properties.

Presented at the 863rd meeting of the AMS, January 18, 1991. 
the $n$-MP are arcs and simple closed curves. From Theorem 3.2 and [7] it follows that no plane continuum can have the $n$-MP for $n>2$. However, a theorem of Bagemihl and Erdös [1] states that, given $n$, there exists a subset of $E^{2}$ with the property that its intersection with every line consists of $n$ points. Such a set has the $n$-MP. Mazurkiewicz [9] used the Axiom of Choice to prove the existence of a subset of $E^{2}$ that meets every line in exactly two points. Larman [3] showed that an $F_{\sigma}$-set $E^{2}$ that meets every line at least once must meet some line at least three times.

Midsets have also been called bisectors [2] or equidistant sets [11, 12], but for subsets of Euclidean spaces it is helpful to distinguish between bisectors and midsets. If $a$ and $b$ are two points of a subset $X$ of $E^{2}$, the bisector $B(a, b)$ of $a$ and $b$ is the straight line that bisects and is perpendicular to the line segment joining $a$ and $b$, while the midset $M(a, b)$ is the intersection of $B(a, b)$ with $X$. Thus, $E^{2}-B(a, b)$ has two components; the one containing the point $a$ is called the a-side of $B(a, b)$.

\section{BASIC LEMMAS AND DEFINITIONS}

A continuum is a compact, connected metric space containing more than one point, and a plane continuum is a continuum that lies in the Euclidean plane $E^{2}$ and has the subspace topology. The standard Euclidean metric $\rho$ is used for $E^{2}$. An arc is a homeomorphic image of the closed interval $[0,1]$ on the real line, while a triod is any homeomorphic image of the union of the closed intervals $[(-1,0),(1,0)]$ and $[(0,0),(0,1)]$ in $E^{2}$. The image $v$ of $(0,0)$ in a triod $T$ is called the vertex of $T$, while the closures of the components of $T-\{v\}$ are called the legs of $T$. A simple closed curve is a homeomorphic image of a circle in $E^{2}$, and a triodic continuum is a continuum containing a triod.

Lemma 2.1. A continuum with the n-MP is locally connected, path connected, and locally path connected.

An indirect proof that a continuum with a finite midset property is locally connected is easily obtained from [10, Theorem 11, p. 90]. For the remainder of Lemma 2.1 see [13, Theorem 31.4].

A connected subset $X$ of $E^{2}$ is said to cross a line (or simple closed curve) $L$ at a point $m \in L$ if there are arcs $A^{\prime}$ and $A^{\prime \prime}$ in $X$ such that $A^{\prime} \cap A^{\prime \prime}=\{m\}$ and $A^{\prime}-\{m\}$ and $A^{\prime \prime}-\{m\}$ lie on opposite sides of $L$. The set $X$ is said to bounce off a line (or simple closed curve) $L$ at a point $m \in L$ if there is an arc $A$ in $X$ such that $m$ lies in the interior of $A, A \cap L=\{m\}$, and $A-\{m\}$ lies in a single component of $E^{2}-L$. Also $X$ is said to bounce off a bisector $B(a, b)$ at $m$ to the a-side of $B(a, b)$ if there is an $\operatorname{arc} A$ in $X$ such that $m \in B(a, b) \cap \operatorname{Int} A$ and $A-\{m\}$ lies on the $a$-side of $B(a, b)$. An arc $A$ is said to hang to the side $S$ of a line $L$ at a point $v \in L$ if $v$ is an end point of $A$ and $(A-\{v\}) \subset S$.

A point $t$ in a continuum $X$ is a bounce point for $X$ if there exist points $x_{t}$ and $y_{t}$ in $X$ such that $t \in B\left(x_{t}, y_{t}\right)$ and $X$ bounces off $B\left(x_{t}, y_{t}\right)$ at $t$. A point $e$ of $X$ is called an end point of $X$ if no arc in $X$ has $e$ in its interior, and a line $L$ is said to be a bisector for $X$ if there exist points $x$ and $y$ of $X$ such that $L=B(a, b)$. 
Lemma 2.2. If a continuum $X$ in $E^{2}$ has the $n-M P$ and $X$ contains at most a finite number of points that are vertices of a triod in $X$, then the set of bounce points for $X$ is countable.

Proof of Lemma 2.2. Suppose the set $W$ of bounce points for $X$ is uncountable. For each $x \in W$ it follows from the $n$-MP that there is an interval $I(x)$ in a bisector for $X$ such that $x$ is the midpoint of $I(x)$ and $I(x) \cap X=\{x\}$. There exists a positive integer $m$ such that the set $W_{m}$ of elements $x$ of $W$ with corresponding interval $I(x)$ of length at least $1 / m$ is infinite, and there must be a sequence $\left\{x_{i}\right\}$ of points in $W_{n}$ such that the corresponding sequence $\left\{I\left(x_{i}\right)\right\}$ of intervals converges to a continuum $H$ [10, Theorems 58 and 59]. Then $H$ is a line segment whose midpoint $p$ lies in $X$. Let $N$ be a neighborhood of $p$ such that $N \cap X$ is path connected (Lemma 2.1) and $N$ contains no end points of any $I\left(x_{i}\right)$, and let $A$ be an arc in $X \cap N$ from a point $x_{j}$ to $p$. Then for infinitely many $k, A$ must cross $I\left(x_{k}\right)$ at the point $x_{k}$. Because $x_{k} \in W_{m}$, there exists an arc $B_{k}$ in $X$ such that $B_{k}$ bounces off the line through $I\left(x_{k}\right)$ at $x_{k}$. Then $A \cup B_{k}$ contains a triod. Since $X$ contains at most a finite number of vertices of triods that lie in $X$, this contradiction establishes Lemma 2.2.

If $X$ is a set with the $n$-MP and $\{x, y\} \subset X$, then a point $c$ in $X \cap B(x, y)$ is called a bad point of $B(x, y)$ if no arc in $X$ crosses $B(x, y)$ at $c$. If $v \in X$, $\beta(v)$ is defined as the collection of all bisectors $B(x, y)$ such that $v \in B(x, y)$, $\{x, y\} \subset X$, and some point of $M(x, y)-\{v\}$ is a bad point of $B(x, y)$.

Frequently used in the sequel, Lemma 2.3 gives three conditions each of which implies the existence of bisectors containing bad points. Proofs for the three parts are easily supplied based on the geometrical fact that if $n-1$ arcs in the continuum $X$ cross a bisector $B(a, b)$ at each of $n-1$ points in $M(a, b)-$ $\{v\}$, which happens if $B(a, b) \notin \beta(v)$, and $X$ contains an arc that bounces off $B(a, b)$ at $v$, then, by moving one of either $a$ or $b$ slightly within $X$, one can produce another bisector that intersects $X$ in at least $n+1$ points, including two points near $v$. Of course this would contradict $X$ having the $n$-MP.

Lemma 2.3. If $X$ is a continuum in $E^{2}, X$ has the $n-M P, C$ is a circle centered at $v, \mathscr{V}$ is a component of $E^{2}-C, a$ and $b$ are points of $C \cap X$, and $X$ contains an arc $A$ that bounces off $B(a, b)$ at the point $v$, then any one of the three following conditions implies $B(a, b) \in \beta(v)$ :

(1) $a$ and $b$ are both limit points of $\mathscr{V} \cap X$;

(2) $a$ is a limit point of both $X \cap \operatorname{Int} C$ and $X \cap \operatorname{Ext} C$; or

(3) $A$ bounces off $B(a, b)$ to the a-side of $B(a, b)$ at $v$, and either $a$ is a limit point of $X \cap \operatorname{Ext} C$ or $b$ is a limit point of $X \cap \operatorname{Int} C$.

While Lemma 2.3 is used to produce bisectors with bad points, Lemma 2.4 limits the number of such bisectors.

Lemma 2.4. If $X$ is a locally path connected subset of $E^{2}$ with the $n$-MP for some positive integer $n$ and $v \in X$, then $\beta(v)$ is a countable collection of lines.

Proof of Lemma 2.4. Suppose $\beta(v)$ is uncountable. Let $\beta(v)=\beta$. For each $B \in \beta$, there exist a point $x$ and an interval $I(x) \subset B$ such that $x$ is the midpoint of $I(x), x$ is a bad point of $B, X \cap I(x)=\{x\}$, and $x \neq v$. Then 
$\beta^{\prime}=\{I(x) \mid B \in \beta\}$ is an uncountable collection of pairwise disjoint intervals, so, as in the proof of Lemma 2.2, there exists an interval $I\left(x_{k}\right) \in \beta^{\prime}$ such that some arc in $X$ crosses $I\left(x_{k}\right)$ at $x_{k}$. This contradicts the choice of $x_{k}$ as a bad point of the bisector in $\beta$ containing $I\left(x_{k}\right)$, and Lemma 2.4 follows.

Lemma 2.5. If $X$ is a continuum in $E^{2}$ that contains an most a finite number of points that are the vertex of a triod in $X, e$ is an end point of an arc $L$ in $X$ such that $e$ is not a limit point of $X-L, C$ is a circle centered at $e, p$ and $q$ are points of $C \cap X, N$ is a neighborhood of $B(p, q)$ such that $N$ contains no vertex of a triod in $X, e$ is the only end point of $X$ or of $L$ that lies in $N$, and either one of the following two properties holds:

(1) $p$ and $q$ are limit points of $\mathscr{V} \cap X$ for some component $\mathscr{V}$ of $E^{2}-C$;

(2) $q$ is a limit point of $\mathscr{V} \cap X$ for each component $\mathscr{V}$ of $E^{2}-C$;

then $X$ cannot have the $n-M P$ for any positive integer $n$.

Proof of Lemma 2.5. Suppose $X$ has the $n$-MP for some positive integer $n$ and the first condition in Lemma 2.5 holds. We may assume $L-\{e\}$ lies on the $p$-side of $B(p, q)$. Since $B(p, q)$ contains no end point of $X$, except for $e$, and $N$ contains no vertex of a triod in $X$, each point of $M(p, q)-\{e\}$ is either a bounce point of $X$ or a point where an arc in $X$ crosses $B(p, q)$-no point of $M(p, q)$ can be both. For each $t \in M(p, q)-\{e\}$, let $A_{t}$ and $B_{t}$ be arcs in $N \cap X$ such that $A_{t} \cap B_{t}=\{t\}$ and $\left(A_{t} \cup B_{t}\right) \cap B(p, q)=\{t\}$. Then $A_{t} \cup B_{t}$ either bounces off or crosses $B(p, q)$ at $t$. Let $F=\left\{H \mid H=A_{t}\right.$ or $H=B_{t}$ for some $\left.t \in M(p, q)-\{e\}\right\}$, and choose open subsets $P$ and $Q$ of $X \cap \mathscr{V}$ whose closures contain $p$ and $q$, respectively, such that $M(x, y) \subset N$ whenever $x \in P \cup\{p\}$ and $y \in Q \cup\{q\}$. By Lemma 2.2 there must exist sequences $\left\{p_{i}\right\}$ and $\left\{q_{i}\right\}$ of points in $P$ and $Q$ that converge to $p$ and $q$, respectively, such that no arc in $X$ bounces off either $B\left(p, q_{i}\right)$ or $B\left(p_{i}, q\right)$ for any $i$. We assume, without loss of generality, that $\mathscr{V}=\operatorname{Ext} C$, from which it follows that for every $i, B\left(p, q_{i}\right) \cap L=\varnothing$, while there is precisely an odd number of points in $B\left(p_{i}, q\right) \cap L$.

By reducing the size of $N$, if necessary, we may assume the only end points of elements of $F$ that are in $N$ are those that belong to $M(p, q)$. There must be an integer $j$ such that, for each $H \in F$, either $B\left(p, q_{j}\right)$ or $B\left(p_{j}, q\right)$ separates the end points of $H$. For $B \in\left\{B\left(p, q_{j}\right), B\left(p_{j}, q\right)\right\}, B$ contains no bounce points of $X$. This means either $B \cap H=\varnothing$ or $B$ intersects $H$ in precisely an odd number of points for each $H \in F$. Let $C R$ be the number of points of $B(p, q)$ where $X$ crosses $B(p, q)$, and let $B O$ be the number of points where $X$ bounces off $B(p, q)$. Then, counting the point $e$ separately, $n=1+C R+B O$. Let $B O_{1}$ be the number of points $t$ of $M(p, q)$ where $B\left(p, q_{j}\right)$ intersects both $A_{t}$ and $B_{t}$, and let $B O_{2}=B O-B O_{1}$. Then $B\left(p, q_{j}\right)$ intersects the arc $A_{t} \cup B_{t}$ an even, possibly zero, number of times whenever $A_{t} \cup B_{t}$ bounces off $B(p, q)$ at $t$. Counting the points in $B\left(p, q_{j}\right) \cap X$, first those near the $C R$ crossing points of $B(p, q)$, we obtain

$$
n=\left\{\sum_{i=1}^{C R+1}\left(2 \alpha_{i}+1\right)\right\}+\left\{\sum_{i=1}^{B O_{1}} 2 \sigma_{i}\right\}=2\left\{\sum_{i=1}^{C R} \alpha_{i}+\sum_{i=1}^{B O_{1}} \sigma_{i}\right\}+C R
$$


On the other hand, the count for $B\left(p_{j}, q\right) \cap X$ yields

$$
n=\left\{\sum_{i=1}^{C R+1}\left(2 \alpha_{i}^{\prime}+1\right)\right\}+\left\{\sum_{i=1}^{B O_{2}} 2 \sigma_{i}^{\prime}\right\}=2\left\{\sum_{i=1}^{C R+1} \alpha_{i}^{\prime}+\sum_{i=1}^{B O_{2}} \sigma_{i}^{\prime}\right\}+C R+1,
$$

where $\left(2 \alpha_{C R+1}^{\prime}+1\right)$ is the odd number of intersections of $B\left(p_{j}, q\right)$ with the arc $L$. Equating these two expressions for $n$, we obtain the contradiction that $n$ is both odd and even.

The proof of the second part of Lemma 2.5, where $q$ is a limit point of both $X \cap \operatorname{Int} C$ and $X \cap \operatorname{Ext} C$, is similar to the above, except that the points $q_{i}$ and $p_{i}$ are selected to both converge to $q$ with $p_{i}$ and $q_{i}$ on opposite sides of $C$. We leave it to the reader to fill in the details to obtain the same contradiction that $n$ is both odd and even. Lemma 2.5 follows.

\section{NO TRIODIC PLANE CONTINUUM HAS THE $n$-MP}

In the following theorem one may assume $n>1$ since it is easy to show (using, e.g., Lemma 2.3) that a triodic continuum cannot have each of its midsets consist of a single point.

Theorem 3.1. If a continuum $X$ in $E^{2}$ has the $n$-MP for some natural number $n$, then $X$ cannot contain a triod.

Proof. Suppose $X$ is a continuum in $E^{2}$ such that $X$ contains triod $\Upsilon$ and $n$ is a natural number such that $X$ has the $n$-MP. Let the vertex of $\Upsilon$ be $v$, and let $W_{i}$, for $i=1,2,3$, denote the legs of $\Upsilon$. For each $r>0$ let $C(r)$ denote the circle of radius $r$ that has its center at $v$, and choose $u>0$ such that $C(u) \cap W_{i} \neq \varnothing$, for each $i$. Then $\Upsilon$ contains a triod $T$ with vertex $v$ and legs $L_{i}$ such that, for $i=1,2,3, L_{i} \subset W_{i}$ and $L_{i}$ has an end point $e_{i}$ such that $L_{i} \cap C(u)=\left\{e_{i}\right\}$. For $p \in E^{2}$, let $C(p)=C(\rho(v, p))$. Although most of the following sixteen numbered assertions are stated relative to the vertex $v$, some of them are later applied as well at other vertices of triods in $X$. The proof of assertion (16), the last one, uses Lemma 2.5 to produce a contradiction.

(1) If, for some $r>0, C(r) \cap X$ contains an arc and $c$ is a point of $C(r) \cap X$, then $c$ cannot be a limit point of both $X \cap \operatorname{Int} C(r)$ and $X \cap \operatorname{Ext} C(r)$.

Proof of (1). Suppose there exists $r>0$, an arc $A \subset C(r) \cap X$, and a point $c \in X \cap C(r)$ such that $c$ is a limit point of both $X \cap \operatorname{Int} C(r)$ and $X \cap \operatorname{Ext} C(r)$. For each $m \in A-\{c\}, X$ must contain an arc that bounces off $B(m, c)$ at $v$. It follows from Lemma 2.3.2 that $B(m, c)$ must contain a bad point. Then the uncountable set $\{B(m, c) \mid m \in A-\{c\}\}$ lies in the set $\beta(v)$, which contradicts Lemma 2.4. (1) follows.

(2) If, for some $r>0, C(r) \cap X$ contains an arc $A$, then the end points of $A$ cannot both be limit points of either $X \cap \operatorname{Int} C$ or $X \cap \operatorname{Ext} C$.

Proof of (2). The proof is much the same as for (1).

Definition. An annulus at a point $v^{\prime}$ is the open annulus between two circles centered at $v^{\prime}$, and an arc $A$ is said to span an annulus $U$ if $\operatorname{Int} A \subset U$ and the end points of $A$ lie in different components of $B d U$.

(3) If $U$ is an annulus at $v$ and $A_{1}, A_{2}$, and $A_{3}$ are disjoint arcs in $X$ such that each $A_{i}$ spans $U$, then, for each circle $C(r)$ lying $U$, each component of $C(r) \cap X$ is a point. 
Proof of (3). Suppose there exists an $r$ such that $C(r) \subset U$ and $C(r) \cap X$ contains an arc. It follows from (1) that, for each $i \in\{1,2,3\}$, there exists an arc $\theta_{i}$ such that $\theta_{i} \subset A_{i} \cap C(r)$ and from (2) that one end point $a_{i}$ of $\theta_{i}$ is a limit point of $L_{i} \cap \operatorname{Int} C(r)$ and the other end point $b_{i}$ of $\theta_{i}$ is a limit point of $L_{i} \cap \operatorname{Ext} C(r)$. If each $\theta_{i}$ is given an orientation from $a_{i}$ to $b_{i}$, then some two of them, say $\theta_{1}$ and $\theta_{2}$, must have the same orientation on $C(r)$, and with no loss in generality, it may be assumed $a_{1} b_{1} a_{2} b_{2}$ is the clockwise orientation on $C(r)$. Let $\omega$ be the clockwise rotation about $v$ such that $\omega\left(a_{1}\right)=a_{2}$, and choose an arc $\Psi$ in $\omega\left(\theta_{1}\right) \cap \theta_{2}$ such that $a_{2} \in \Psi$. For each $x \in \Psi, B\left(a_{1}, x\right)$ separates $a_{1}$ from $a_{2}$ and there exists an arc in $T$ that bounces off $B\left(a_{1}, x\right)$ at $v$. For $i \in\{1,2\}$, define $\Psi_{i}=\left\{x \in \Psi\right.$ : there is an $\operatorname{arc} A_{x} \subset T$ such that $A_{x}$ bounces off $B\left(a_{1}, x\right)$ at $v$ to the $a_{i}$-side of $\left.B\left(a_{1}, x\right)\right\}$.

For each $x \in \Psi_{2}$, it follows from Lemma 2.3.3 that $B\left(a_{1}, x\right)$ contains a bad point. This means $\Psi_{2}$ is countable because $\beta(v)$ is countable (Lemma 2.4). Since $\Psi=\Psi_{1} \cup \Psi_{2}$ and $\Psi$ is an arc, $\Psi_{1}$ must be uncountable. For each $x \in \Psi_{1}$, the point $\omega^{-1}(x)=x^{\prime} \in \theta_{1}$ has the property $B\left(a_{1}, x\right)=B\left(x^{\prime}, a_{2}\right)$. But $a_{2}$ is a limit point of $X \cap \operatorname{Int} C(r)$, so $B\left(x^{\prime}, a_{2}\right)$ contains a bad point by Lemma 2.3.3. This contradicts Lemma 2.4 because the set $\left\{B\left(a_{1}, x\right) \mid x \in\right.$ $\left.\Psi_{1}\right\}=\left\{B\left(x^{\prime}, a_{2}\right) \mid x^{\prime} \in \omega^{-1}\left(\Psi_{1}\right)\right\}$ is uncountable. (3) follows.

(4) If $r \in(0, u)$, then each component of $C(r) \cap X$ is a point.

Proof of (4). (4) is an immediate consequence of (3) and the fact that each $L_{i}$ spans the open annulus $C(u)-\{v\}$.

(5) If $X$ contains a triod $T^{\prime}$ with vertex $v^{\prime}, U$ is an annulus at $v^{\prime}$, and there exist arcs $A_{1}, A_{2}, A_{3}$ in $X$, each of which spans $U$, such that for every $x \in A_{1}$, points $y \in A_{2}$ and $z \in A_{3}$ exist with the following properties:

(a) $\rho\left(v^{\prime}, x\right)=\rho\left(v^{\prime}, y\right)=\rho\left(v^{\prime}, z\right)$ and

(b) $\{B(x, y), B(y, z), B(x, z)\} \subset B\left(v^{\prime}\right)$,

then there exists an annulus $U^{\prime}$ in $U$ at $v^{\prime}$ and three $\operatorname{arcs} X_{1}, X_{2}, X_{3}$ such that, for each $i, X_{i} \subset A_{i}, X_{i}$ spans $U^{\prime}$, and $X_{i} \cup\left\{v^{\prime}\right\}$ lies in a straight line.

Proof of (5). Since $\beta\left(v^{\prime}\right)$ is countable by Lemma 2.4, the collection $\beta^{\prime}$ of all ordered triples of elements of $\beta\left(v^{\prime}\right)$ is also countable. Let $T_{i}, i=1,2,3, \ldots$, denote the elements of $\beta^{\prime}$, and for each $i$, define $M_{i}$ to be the set of all points $x \in A_{1}$ such that there exist $y \in A_{2}$ and $z \in A_{3}$ with $(B(x, y), B(y, z)$, $B(x, z))=T_{i}$. By hypothesis, $A_{1}=\bigcup_{i=1}^{\infty} M_{i}$, and it is not difficult to prove that each $M_{i}$ is closed. A Baire category theorem [11, 25, p. 185] shows the existence of an integer $k$ and an arc $X_{1}^{\prime}$ in $A_{1}$ such that $X_{1}^{\prime} \subset M_{k}$. Since $v^{\prime}$ is the vertex of a triod in $X$, (3) can be applied at the vertex $v^{\prime}$ to see that $X_{1}^{\prime}$ cannot lie in a circle at $v^{\prime}$. This means there exists an open annulus $U^{\prime}$ in $U$ at $v^{\prime}$ and a subarc $X_{1}$ of $X_{1}^{\prime}$ such that $X_{1}$ spans $U^{\prime}$. Let $\left(B_{1}, B_{2}, B_{3}\right)=T_{k}$, and let $R_{i}$, for $i \in\{1,2,3\}$, be the reflection of $E^{2}$ in $B_{i}$. If the composition $R_{2} \circ R_{1}$ is denoted by $\omega$, a rotation about $v^{\prime}$, then $R_{3} R_{2} R_{1}(x)=R_{3} \omega(x)$ for each $x \in X_{1}$. However, the reflection $R_{3}$ changes the orientation of three noncollinear points while $\omega$ does not. Since the composition $R_{3} \circ \omega$ is the identity map on $X_{1}$, the point set $X_{1} \cup\left\{v^{\prime}\right\}$ must be collinear. To complete (5), let $X_{2}=R_{1}\left(X_{1}\right)$ and $X_{3}=R_{2}\left(X_{2}\right)$.

(6) If $0<t<r \leq u, L$ is an arc in $X$ from $v$ to a point $e \in C(r)$, and $L \subset C(r) \cup$ Int $C(r)$, then $L \cap C(t)$ contains no more than two points. 
Proof of (6). Suppose there exist an arc $L$ as in (6) and a circle $C(t), 0<t<r$, such that $C(r) \cap L$ contains three points. Impose an order on $L$ with $v$ as the first point. Since $t<u$ it follows from (4) that each component of $L \cap C(t)$ is a point, so an annulus $U$ exists at $v$ such that $L$ contains three disjoint arcs $A_{1}, A_{2}, A_{3}$ each spanning $U$ and $U \subset \operatorname{Int} C(r)$. Assume $A_{1}<A_{2}<A_{3}$ in the order on $L$, and assume further that if $A$ is an arc in $L$ that also spans $U$ such that $A \neq A_{i}$, for $i \in\{1,2\}$, then $A_{2}<A$ on $L$. From (4) and Lemma 2.3.1 one sees that (5) applies, and from (5), it may be assumed, without loss of generality, that each of the sets $A_{i} \cup\{v\}$ lies in a straight line. For each $i$, let $B d A_{i}=\left\{a_{i}, b_{i}\right\}$ where $a_{i}<b_{i}$ in the order on $L$. This means $b_{2}$ lies between $v$ and $a_{2}$ on the line $B$ through $A_{2} \cup\{v\}$. If $x<y$ in $L$ the interval notation $[x, y]$ is used to denote the subarc of $L$ joining $x$ to $y$.

Let $R$ be the reflection of the plane in $B$, let $K$ be the component of $E^{2}-\left(\left[v, a_{2}\right] \subset R\left(\left[v, a_{2}\right]\right)\right)$ containing $b_{2}$, and note that $K \subset \operatorname{Int} C(r)$. Since $b_{2} \in K \subset$ Int $C(r)$ and $e \in C(r)$, the arc $\left[b_{2}, e\right]$ intersects $B d K$ at some point $x$ in $R\left(\left(v, a_{2}\right)\right)$. Then $B\left(x, R^{-1}(x)\right)=B$, which contradicts the $n$-MP since $A_{2} \subset B$. Then (6) follows.

(7) For $i \in\{1,2,3\}$ and $t \in(0, u], C(t) \cap L_{i}$ is a point.

Proof of (7)w. Suppose (7) false. By construction $C(u) \cap L_{i}$ is a point for each $i$, so there exist $t \in(0, u)$ and $j \in\{1,2,3\}$ such that $L_{j} \cap C(t)$ contains two points. But since $C(t)$ separates the end points of $L_{j}$, it follows from (4) that there must exist $t^{\prime}$ near $t$ such that $L_{j} \cap C\left(t^{\prime}\right)$ contains three points. This contradicts (6), and (7) follows.

(8) For $i \in\{1,2,3\}, L_{i}$ is a straight line segment.

Proof of (8). From Lemma 2.3 and (7) it follows that each bisector $B(x, y)$, where $x$ and $y$ are chosen equidistant from $v$ and in different legs of $T$, lies in $\beta(v)$. From (7), the collection $F_{12}=\left\{B(x, y) \mid x \in L_{1}, y \in L_{2}\right.$, $\{x, y\} \subset C(r)$, and $0<r \leq u\}$ is a continuous family of lines in $\beta(v)$. If $F_{12}$ contains two distinct bisectors, then it contains uncountably many between them. Therefore, since $\beta(v)$ is countable by Lemma 2.4, $F_{12}$ consists of a single line $B_{1}$. The analogous sets $F_{23}$ and $F_{13}$ similarly consist of single lines $B_{2}$ and $B_{3}$, respectively. As in the last part of the proof of (5), this means $L_{1}$ transposes to itself under the composition of a rotation about $v$ and a reflection in $B_{3}$. Unless $L_{1}$ is a line segment, this is a contradiction, and similarly, $L_{2}$ and $L_{3}$ must be segments. From this, (8) follows.

Definitions and Notation. With $T$ fixed, let $\Gamma_{T}=\{u \mid C(u)$ meets each of the three legs of a triod containing $T$ in precisely one point $\}$. Since $X$ is compact, $\Gamma_{T}$ has a least upper bound $\mu_{T}$, and it follows from (8) that there exists a triod $T^{*}$ in $X \cap\left(C\left(\mu_{T}\right) \cup \operatorname{Int} C\left(\mu_{T}\right)\right)$ such that $T \subset T^{*}$ and the legs of $T^{*}$ lie on lines through $v$. Now let $T$ vary; define $\Gamma=\left\{\mu_{T} \mid T\right.$ is a triod in $X \cap\left(C\left(\mu_{T}\right) \cup \operatorname{Int} C\left(\mu_{T}\right)\right)$ with vertex $\left.v\right\}$, and let $\mu$ be the least upper bound of $\Gamma$. To see there is a triod $T^{* *}$ in $X \cap(C(\mu) \cup \operatorname{Int} C(\mu))$ with $v$ as its vertex, suppose not. Then there must be an infinite sequence $\left\{I_{i}\right\}$ of straight line segments in $X \cap(C(\mu) \cup \operatorname{Int} C(\mu))$ such that $v$ is one end point of each $I_{i}$ and diam $I_{i}$ \} converges to $\mu$. Since $X$ is compact, some subsequence of $\left\{I_{i}\right\}$ converges [10, Theorems 58 and 59] to an interval $I$ that also must lie in 
$X$. But then $M(v, x)$, where $v$ and $x$ are the end points of $I$, must contain infinitely many points of $X$, contrary to the $n$-MP.

In the sequel, let $C$ denote the "maximal" circle $C(\mu)$, let $T$ denote the "maximal" triod $T^{* *}$, and for each $i$, let $L_{i}$ denote a leg of $T$. For $i \in$ $\{1,2,3\}$, let $\theta_{i}$ denote the component of $X \cap C$ containing the end point $e_{i}$ of the line segment $L_{i}$. From the choices of $C$ and $T$, it follows from Lemma 2.1 that not all three of the sets $\theta_{i}$ can contain limit points of $X \cap \operatorname{Ext} C$.

(9) The legs of $T$ may be named such that $\theta_{1}=\left\{e_{1}\right\}$ and $\theta_{2}=\left\{e_{2}\right\}$.

Proof of (9). Suppose two of the sets, say $\theta_{1}$ and $\theta_{2}$, contain more than one point. Without loss of generality, assume the arc $L_{2} \cup L_{3}$ bounces off $B\left(e_{1}, e_{2}\right)$ to the $e_{2}$-side of $B\left(e_{1}, e_{2}\right)$ at $v$. Then, because of $(8)$, there is a subarc $\theta_{2}^{\prime}$ of $\theta_{2}$ such that $L_{2} \cup L_{3}$ bounces off $B\left(e_{1}, x\right)$ to the $x$-side of $B\left(e_{1}, x\right)$ at $v$ for all $x$ in $\theta_{2}^{\prime}$. Because $e_{1}$ is a limit point of $X \cap \operatorname{Int} C$, it follows from Lemma 2.3.3 that $B\left(e_{1}, x\right) \in \beta(v)$ for every $x \in \theta_{2}^{\prime}$. But this contradicts Lemma 2.4, and (9) follows.

(10) Without loss of generality, $e_{1}$ is not a limit point of $X \cap(C \cup \operatorname{Ext} C)$.

Proof of (10). By the choice of $T$ and $C$ as the maximal triod and circle at $v$, respectively, (see the remarks following the proof of (8)) one of the three points $e_{1}, e_{2}$, and $e_{3}$, say $x$, is not a limit point of $X \cap \operatorname{Ext} C$. If $x$ cannot be chosen to be $e_{1}$, then $e_{1}$ is a limit point of $X \cap$ Ext $C$ and it follows from (1) that $\theta_{3}=\left\{e_{3}\right\}$. Then $x$ cannot be a limit point of $X \cap C$. In this case rename the legs of $T$ and their end points such that $x=e_{1}$, and (10) follows.

(11) The point $e_{1}$ is not a limit point of $X-L_{1}$.

Proof of (11). Suppose (11) is false. Then by (10), $e_{1}$ is a limit point of $X \cap$ Int $C-L_{1}$. Let $A$ be an arc in $X$ joining $e_{1}$ to a point $f \in \operatorname{Int} C$ such that $A \cap L_{2}=\varnothing$ and $f \notin L_{1}$. By (10), we may assume $A-\left\{e_{1}\right\} \subset \operatorname{Int} C$. For each $a \in A$, let $a^{\prime}$ be the unique point of $L_{2} \cap C(a)$, and note that, by Lemma 2.3.2, $B\left(a, a^{\prime}\right) \in \beta(v)$, for each $a \in A-\left\{e_{1}\right\}$. Also note that $e_{1}^{\prime}=e_{2}$; and $B\left(e_{1}, e_{1}^{\prime}\right) \in \beta(v)$ by Lemma 2.3.1. Since every line between $B\left(e_{1}, e_{1}^{\prime}\right)$ and $B\left(f, f^{\prime}\right)$ is a bisector $B\left(a, a^{\prime}\right)$ for some point $a \in A$, the set $\left\{B\left(a, a^{\prime}\right) \mid a \in A\right\}$ is an uncountable subset of $\beta(v)$. But this contradicts Lemma 2.4, and (11) follows.

(12) If $T^{\prime}$ is a triod in $X \cap(C \cup \operatorname{Int} C)$ and $v^{\prime}$ is the vertex of $T^{\prime}$, then $v=v^{\prime}$.

Proof of (12). Suppose $X$ contains a triod $T^{\prime}$ as in the statement of (12) such that $v^{\prime} \neq v$. As with $T$, it follows from (8) that the legs of $T^{\prime}$ are line segments, so the interiors of all three legs of $T^{\prime}$ lie in Int $C$. Furthermore, at least one of the three legs of $T^{\prime}$, say $G$, is not linear with the point $v$. Then there is an open annulus $U$ at $v$ and three disjoint arcs $A_{1} \subset L_{1}, A_{2} \subset L_{2}$, and $A_{3} \subset G$ that span $U$ such that $U \subset C \cup \operatorname{Int} C$. Applying (5) at the point $v$, we obtain the contradiction that the subsegment $A_{3}$ of $G$ lies on a line through $v$. (12) follows.

(13) If $v^{\prime}$ is a vertex of a triod in $X$, then there is a circle $C^{\prime}$ at $v^{\prime}$ and a triod $T^{\prime}$ in $X$ with vertex $v^{\prime}$ and legs $L_{1}^{\prime}, L_{2}^{\prime}, L_{3}^{\prime}$ such that:

(a) for each $i \in\{1,2,3\} . L_{i}^{\prime} \subset C^{\prime} \cup \operatorname{Int} C^{\prime}$ and $L_{i}^{\prime} \cap C^{\prime} \neq \varnothing$; 
(b) each of $L_{1}^{\prime} \cap C^{\prime}$ and $L_{2}^{\prime} \cap C^{\prime}$ is a point;

(c) no point of $L_{1}^{\prime}-\left\{v^{\prime}\right\}$ is a limit point of $X-L_{1}^{\prime}$; and

(d) the component of $X \cap\left(C^{\prime} \cup \operatorname{Int} C^{\prime}\right)$ containing $v^{\prime}$ is the union of finitely many line segments each with $v^{\prime}$ as an end point.

Proof of (13). Since $v$ was chosen as an arbitrary vertex of a triod in $X$, it suffices to show that the above properties hold with the primes removed. The first property in (13) is clear, the second follows from (9), and the third from Lemma 2.1, (11), and (12). From (8), the component of $X \cap(C \cup \operatorname{Int} C)$ containing $v$ is the union of line segments. Suppose there are infinitely many line segments in $X \cap(C \cup \operatorname{Int} C)$, each with $v$ as an end point. Then say infinitely many such segments lie on the $e_{2}$-side of $B\left(e_{2}, e_{3}\right)$, and a point $e_{3}^{\prime}$ can be chosen in $L_{3}$ close enough to $e_{3}$ that $B\left(e_{2}, e_{3}^{\prime}\right)$ intersects more than $n$ of these segments near $v$. This contradicts the $n$-MP, and the fourth property of (13) follows.

(14) There are at most two points of $X$ that are vertices of a triod in $X$.

Proof of (14). Suppose that in addition to $T$ there are two triods $T^{\prime}$ and $T^{\prime \prime}$ with distinct vertices $v^{\prime}$ and $v^{\prime \prime}$ neither of which is $v$. By (8) it may be assumed that the legs of all three triods are straight line segments. Without loss of generality, assume $\rho\left(v, v^{\prime}\right) \leq \rho\left(v, v^{\prime \prime}\right)$, and to obtain a contradiction, suppose $\rho\left(v, v^{\prime}\right)=\rho\left(v, v^{\prime \prime}\right)$. On one side of $C\left(\rho\left(v, v^{\prime}\right)\right)$ there is an open annulus $U$ at $v$ and three segments in legs of $T^{\prime} \cup T^{\prime \prime}$ that span $U$. Then from (5) these three segments, and hence the legs that contain them, are collinear with $v$. Since no two legs of either $T^{\prime}$ or $T^{\prime \prime}$ can be collinear with $v$, this is a contradiction. Thus $\rho\left(v, v^{\prime}\right)<\rho\left(v, v^{\prime \prime}\right)$ and similarly, $\rho\left(v^{\prime}, v^{\prime \prime}\right) \neq \rho\left(v^{\prime}, v\right)$.

Let $A$ be an arc in $X$ from $v$ to $v^{\prime \prime}$. Suppose $v^{\prime} \notin A$. Then there exists an open annulus at $v$ on one side of $C\left(\rho\left(v, v^{\prime}\right)\right)$ that is spanned by three arcs, two lying in legs of $T^{\prime}$ and one in $A$. By (5), two legs of $T^{\prime}$ are collinear with $v$, a contradiction. This means $v^{\prime} \in A$.

Suppose $\rho\left(v^{\prime}, v^{\prime \prime}\right)<\rho\left(v^{\prime}, v\right)$. Let $K$ be the circle at $v^{\prime}$ of radius $\rho\left(v^{\prime}, v^{\prime \prime}\right)$. Then for one side $S$ of $K$, circles concentric with $K$ and in $S$ all intersect $A$ and two legs of $T^{\prime \prime}$. By (5) these two legs of $T^{\prime \prime}$ are collinear with $v^{\prime}$. A similar contradiction results if $\rho\left(v^{\prime}, v^{\prime \prime}\right)>\rho\left(v^{\prime}, v\right)$, and (14) follows.

(15) The continuum $X$ has only finitely many end points.

Proof of (15). Suppose $\left\{d_{i}\right\}$ is an infinite sequence of distinct end points of $X$, and for each $i$, let $A_{i}$ be an arc in $X$ from $v$ to $d_{i}$. By (13), applied at $v$, there must be a line segment in $X$ from $v$ to a point of $C$ that lies in infinitely many of the arcs $A_{i}$. Since no $A_{i}$ contains two end points of $X$, this means there is a triod in $X$ with its vertex $v^{\prime}$ different from $v$. From (14), the point $v^{\prime}$ must be the intersection of infinitely many otherwise disjoint arcs in $X$. But this contradicts (13)(d) when (13) is applied at the point $v^{\prime}$. Then (15) follows.

(16) $X$ cannot have the $n$-MP.

Proof of (16). There must exist points $p$ and $q$ in $T-\{v\}$ such that $B(p, q)$ contains the point $e_{1}$. To see that $p$ and $q$ can also be chosen such that no end point of $X$, except $e_{1}$, lies in $B(p, q)$ and no vertex of a triod in $X$ lies in $B(p, q)$, consider the collection of circles $K(r)$ of radius $r$ centered at 
$e_{1}$. There must exist positive numbers $s<t$ and two legs $L$ and $M$ of $T$ such that $K(r)$ intersects $L$ and $M$ at points $p(r)$ and $q(r)$, respectively, if $s<r<t$. Since that is no $r$ such that $L_{1}$ lies in $B(p(r), q(r))$, it is clear that $\{B(p(r), q(r)) \mid r \in(s, t)\}$ contains an uncountable set of bisectors for $X$. From (14) and (15) the union $E$ of the end points of $X$ with vertices of triods in $X$ is a finite set; so clearly points $p$ and $q$ exist as claimed. Let $N$ be a neighborhood of $B(p, q)$ such that $N \cap E-\left\{e_{1}\right\}=\varnothing$, and observe that, with the use of (11), Lemma 2.5(1) follows, with $K(r)=C$, since both $L$ and $M$ are segments. Then Lemma 2.5 applies to yield a contradiction. This completes (16), and Theorem 3.1 follows.

Theorem 3.2. If a continuum in the Euclidean plane has the $n-M P$ for $n \geq 1$, then it must either be a simple closed curve or an arc.

Since a locally connected continuum is either an arc, a simple closed curve, or is triodic [10, Theorem 75, p. 218], Theorem 3.2 follows from Lemma 2.1 and Theorem 3.1. In [7] we use the results here to prove the stronger theorem that if a planar continuum $X$ has the $n$-MP for $n \geq 1$, then either $n=1$ and $X$ is an arc, or $n=2$ and $X$ is a simple closed curve. With a few additional pages it would be possible to include a proof here that no arc or simple closed curve has the $n$-MP for $n$ an odd integer greater than 1, but the odd and even cases are done together in [7].

\section{REFERENCES}

1. F. Bagemihl and P. Erdös, Intersections of prescribed power, type, or measure, Fund. Math. 41 (1954), 57-67.

2. A. D. Berard, Jr. and W. Nitka, A new definition of the circle by use of bisectors, Fund. Math. 85 (1974), 49-55.

3. D. G. Larman, A problem of incidence, J. London Math. Soc. (2) 43 (1968), 407-409.

4. L. D. Loveland, The double midset conjecture for continua in the plane, Topology Appl. 40 (1991), 117-129.

5. _ No continuum in $E^{2}$ has the TMP; I. Arcs and spheres, Proc. Amer. Math. Soc. 110 (1990), 1119-1128.

6. _ No continuum in $E^{2}$ has the TMP; II. Triodic continua, Rocky Mountain J. Math. (to appear).

7. L. D. Loveland and S. M. Loveland, Finite equidistant sets in plane continua, submitted.

8. L. D. Loveland and S. G. Wayment, Characterizing a curve with the double midset property, Amer. Math. Monthly 81 (1974), 1003-1006.

9. S. Mazurkiewicz, Sur un ensemble plan qui a avec chaque droite deux et seulement deux points communs, C. R. Sci. et Lett. de Varsovie 7 (1914), 382-383.

10. R. L. Moore, Foundations of point set theory, revised edition, Amer. Math. Soc. Colloq. Publ., vol. 13, Amer. Math. Soc. Providence, RI, 1962.

11. Sam B. Nadler, Jr., An embedding theorem for certain spaces with an equidistant property, Proc. Amer. Math. Soc. 59 (1976), 179-183.

12. J. B. Wilker, Equidistant sets and their connectivity properties, Proc. Amer. Math. Soc. 47 (1975), 446-452.

13. Stephen Willard, General topoogy, Addison-Wesley, Reading, MA, 1970.

Department of Mathematics, Utah State University, Logan, Utah 84322-3900

E-mail address: LDL@cc.usu.edu

Department of Mathematics, Weber State University, Ogden, Utah 84408-1702 